\title{
User requirements for future urban vehicles
}

Matthias Klötzke, G. Kopp, S. Schmid, H. E. Friedrich

FK, Deutsches Zentrum für Luft- und Raumfahrt e.V. (DLR)

This manuscript is not available according to publishing restriction. Thank you for your understanding. 